\title{
Revisión teórica sobre los efectos de la actividad física en el adulto mayor
}

\section{Theoretical review on the effects of physical activity on the older adult}

\author{
Daniel Fernando Barrera-Rodríguez ${ }^{1}$
}

'Universidad de Ciencias Aplicadas y Ambientales, U.D.C.A. Bogotá, D.C. danbarrera@udca.edu.co

Cómo citar: Barrera-Rodríguez, D.F. 2021. Revisión teórica sobre los efectos de la actividad física en el adulto mayor Rev. Digit. Act. Fis. Deport. 7(2):e1937. http://doi.org/10.31910/rdafd.v7.n2.2021.1937

Artículo de acceso abierto publicado por Revista Digital: Actividad Física y Deporte, bajo una licencia Creative Commons CC BY-NC 4.0

Publicación oficial de la Universidad de Ciencias Aplicadas y Ambientales U.D.C.A, Institución de Educación Superior Acreditada de Alta Calidad por el Ministerio de Educación Nacional.

Recibido: febrero 21 de 2021 Aceptado: marzo 29 de 2021 Editado por: Álvaro José Gracia Díaz

\section{RESUMEN}

Introducción: Todos los cambios que adopta el adulto mayor son producto de las modificaciones fisiológicas, que se acompañan con la edad. El ejercicio físico, si bien no evita los cambios, retrasa su aparición, disminuye su progresión y su impacto en la salud; todo ello, mejora la calidad y el estilo de vida del adulto mayor. Objetivo: Este artículo de revisión pretende dar una visión sintética, pero precisa, acerca de la actividad física realizada por el adulto mayor, beneficios y efectos producidos por esta. El envejecimiento es un proceso natural, al cual, están sometidos la mayoría de los seres vivos y se ha caracterizado por su acelerado ritmo, acompañado, además, de crecientes procesos de urbanización y de globalización, asociados con un incremento significativo de la inactividad física, que contribuye al desarrollo de enfermedades crónicas no transmisibles, que representan la principal causa de muerte, a nivel global. Metodología: Se hace una revisión de literatura especializada, donde se compilan los estudios de mayor relevancia, que abordan los temas de envejecimiento, salud, adulto mayor y actividad física; a partir de ello, se desarrolla una tabla, donde se describen las metodologías utilizadas en los diversos estudios y otra donde se muestran las conclusiones de dichos estudios. Resultados: Para el adulto mayor, la actividad física desempeña un papel importante en la consecución de un envejecimiento exitoso e integral (físico, psicológico, biológico, emocional y social). Conclusión: Se demuestra los efectos causados por la práctica frecuente de actividad física realizada por el adulto mayor.

Palabras clave: Actividad física; Adulto mayor; Envejecimiento; Salud.

\section{ABSTRACT}

Introduction: All the changes that the elderly adult adopts are the product of the physiological modifications that are accompanied by age; Physical exercise, although it does not prevent changes, delays their onset, slows their progression and their impact on health; All of this improves the quality and lifestyle of the elderly. Objective: This review article tries to give a synthetic, but precise vision about the physical activity carried out by the elderly, benefits and effects produced by it; aging is a natural process to which most living beings are subjected, the aging process has been characterized by its accelerated pace and has also been accompanied by increasing 
urbanization and globalization processes, which have been associated with an increase significant of physical inactivity, which contributes to the development of chronic non-communicable diseases that represent the main cause of death worldwide. Methodology: A review of specialized literature is made, where the most relevant studies that address the issues of aging, health, the elderly and physical activity are compiled, based on this, a table is developed describing the methodologies used in the various studies and another where the conclusions of these studies are shown. Results: For the elderly, physical activity plays an important role in achieving successful and comprehensive aging (physical, psychological, biological, emotional and social). Conclusion: The effects caused by the frequent practice of physical activity carried out by the elderly are demonstrated.

Keywords: Physical activity; Older adult; Aging; Health.

\section{INTRODUCCIÓN}

Para el correcto desarrollo de la presente investigación, se hace una revisión exhaustiva acerca de la temática, los resultados que han generado los autores sobre el tema y las conclusiones, donde se demuestre la relación entre actividad física y envejecimiento. Dulcey-Ruíz (2010) describe el envejecimiento como un fenómeno que se presenta a lo largo de toda la vida, desde el nacimiento hasta la muerte; sin embargo, a pesar de ser un hecho conocido por todos, es difícil de aceptar, como una realidad.

La actividad física es otro término para referirse a aquellas actividades que se realizan en la vida cotidiana. La Organización Panamericana de la Salud y la Organización Mundial de la Salud (OMS, 2002), la refiere como "la gama amplia de actividades y movimientos que incluyen actividades cotidianas, tales como caminar en forma regular y rítmica, jardinería, tareas domésticas pesadas y baile".

Para Jacoby et al. (2003), la inactividad física es un problema relevante en América Latina, especialmente, en la población mayor. Según la Organización Panamericana de la Salud (OPS, 2002), casi las tres cuartas partes de la población adulta es sedentaria, siendo las personas de bajo nivel socioeconómico, las mujeres y los adultos mayores, las más inactivas, durante el tiempo libre o recreación, citado por Gómez et al. (2004) y Monteiro et al. (2003). Adicionalmente, una proporción considerable de la mortalidad ocasionada por ECNT (Enfermedad Crónica No Transmisible), en Colombia, se puede atribuir a los efectos de la inactividad física (Lobelo, 2006).

Con el desarrollo del artículo, se pretende demostrar los beneficios que conlleva la práctica de actividad física sobre los procesos de envejecimiento, de esta manera, se potencia la práctica de actividad física en los adultos mayores. Para Hernández et al. (2010), los beneficios de la actividad física sobre la salud han sido ampliamente documentados. En adultos mayores, la actividad física contribuye a mejorar la flexibilidad, la fuerza, la coordinación y el equilibrio, que propician mayor estabilidad postural, reduciendo el riesgo de caídas y fracturas. Adicionalmente, la actividad física mejora la autoestima, retrasa el deterioro cognitivo, alivia los síntomas de depresión y propicia una mayor integración social. Las personas mayores que realizan actividad física tienen, además, mayor probabilidad de estar integradas a una red de apoyo social.

Basilio (2013) describe otros beneficios de la actividad física en los adultos mayores son emocionales, mejora la actividad mental, la coordinación y el equilibrio, combate el estrés y la ansiedad, provee un mejor estilo de vida, aporta diversión, incide en el estado de ánimo, permite a las personas sentirse útiles e independientes, ayuda a combatir el insomnio, salud en general, disminuye el riesgo de desarrollar enfermedades, interviene en el buen funcionamiento cardiovascular, minimiza la pérdida de masa muscular y ósea, aumenta la flexibilidad, mejora la coordinación motriz y ayuda a mantener el peso.

Todos los cambios que adopta el adulto mayor son producto de modificaciones fisiológicas, que se acompañan con la edad; el ejercicio físico, si bien no evita los cambios, retrasa su aparición, disminuye su progresión y su impacto en la salud; todo ello mejora la calidad y estilo de vida del adulto mayor.

Beneficios y efectos esperados. El propósito del presente artículo es mostrar una perspectiva de la actividad física con la población del adulto mayor, así como los beneficios y los efectos que se obtienen, considerando variables fisiológicas, psicológicas, motrices y sociales; se espera obtener, como resultado, la identificación y la relación que existe 
entre la práctica de actividad física y la salud de los adultos mayores.

Enfoque. La investigación, se categoriza dentro del enfoque mixto, porque se obtiene información cuantitativa y cualitativa (Hernández Sampieri et al. 2014).

Diseño. Es un diseño de tipo transversal, se hace la revisión de los documentos en un tiempo determinado y no se hace una comparación futura dentro del documento (Hernández Sampieri et al. 2014).

Alcance investigativo. El alcance, se define de tipo explicativo, debido a que se encuentra una cantidad considerable de información, haciendo la salvedad de que en el medio local, regional y nacional, el número de investigaciones publicadas llega a ser considerablemente reducido (Hernández Sampieri et al. 2014).

Tabla 1. Resultados de la búsqueda en las bases de datos.

\begin{tabular}{|c|c|c|c|c|c|c|c|}
\hline $\begin{array}{c}\text { Base de } \\
\text { Datos }\end{array}$ & $\begin{array}{c}\text { Ecuaciones de } \\
\text { búsqueda }\end{array}$ & Artículos & Funcional & Loc & Reg & $\mathrm{Nac}$ & Int \\
\hline \multirow[t]{4}{*}{ Scopus: } & $\begin{array}{l}\text { Actividad física + } \\
\text { adultos mayores }\end{array}$ & 80 & 2 & \multirow{4}{*}{0} & \multirow{4}{*}{0} & \multirow{4}{*}{1} & \multirow{4}{*}{4} \\
\hline & $\begin{array}{l}\text { Adulto mayor + } \\
\text { Salud }\end{array}$ & 4 & 1 & & & & \\
\hline & $\begin{array}{l}\text { Actividad física + } \\
\text { Envejecimiento }\end{array}$ & 37 & 0 & & & & \\
\hline & $\begin{array}{l}\text { Actividad física + } \\
\text { Salud }\end{array}$ & 57 & 0 & & & & \\
\hline \multirow[t]{4}{*}{ ClinicalKey: } & $\begin{array}{l}\text { Actividad física }+ \\
\text { adultos mayores }\end{array}$ & 20 & 0 & \multirow{4}{*}{0} & \multirow{4}{*}{0} & \multirow{4}{*}{0} & \multirow{4}{*}{2} \\
\hline & $\begin{array}{l}\text { Adulto mayor + } \\
\text { Salud }\end{array}$ & 18 & 0 & & & & \\
\hline & $\begin{array}{l}\text { Actividad física }+ \\
\text { Envejecimiento }\end{array}$ & 9 & 0 & & & & \\
\hline & $\begin{array}{l}\text { Actividad física + } \\
\text { Salud }\end{array}$ & 14 & 0 & & & & \\
\hline \multirow[t]{4}{*}{$\begin{array}{l}\text { Access } \\
\text { Medicine: }\end{array}$} & $\begin{array}{l}\text { Actividad física + } \\
\text { adultos mayores }\end{array}$ & 22 & 1 & \multirow{4}{*}{0} & \multirow{4}{*}{0} & \multirow{4}{*}{0} & \multirow{4}{*}{1} \\
\hline & $\begin{array}{l}\text { Adulto mayor + } \\
\text { Salud }\end{array}$ & 15 & 0 & & & & \\
\hline & $\begin{array}{l}\text { Actividad física + } \\
\text { Envejecimiento }\end{array}$ & 19 & 0 & & & & \\
\hline & $\begin{array}{l}\text { Actividad física + } \\
\text { Salud }\end{array}$ & 14 & 0 & & & & \\
\hline \multirow[t]{4}{*}{ ProQuest: } & $\begin{array}{l}\text { Actividad física + } \\
\text { adultos mayores }\end{array}$ & 112 & 4 & \multirow{4}{*}{0} & \multirow{4}{*}{1} & \multirow{4}{*}{2} & \multirow{4}{*}{4} \\
\hline & $\begin{array}{l}\text { Adulto mayor + } \\
\text { Salud }\end{array}$ & 90 & 2 & & & & \\
\hline & $\begin{array}{l}\text { Actividad física + } \\
\text { Envejecimiento }\end{array}$ & 27 & 0 & & & & \\
\hline & $\begin{array}{l}\text { Actividad física + } \\
\text { Salud }\end{array}$ & 15 & 0 & & & & \\
\hline \multirow[t]{4}{*}{ ScienceDirect: } & $\begin{array}{l}\text { Actividad física + } \\
\text { adultos mayores }\end{array}$ & 42 & 2 & \multirow{4}{*}{0} & \multirow{4}{*}{0} & \multirow{4}{*}{0} & \multirow{4}{*}{4} \\
\hline & $\begin{array}{l}\text { Adulto mayor + } \\
\text { Salud }\end{array}$ & 34 & 0 & & & & \\
\hline & $\begin{array}{l}\text { Actividad física + } \\
\text { Envejecimiento }\end{array}$ & 43 & 2 & & & & \\
\hline & $\begin{array}{l}\text { Actividad física + } \\
\text { Salud }\end{array}$ & 21 & 0 & & & & \\
\hline \multirow[t]{4}{*}{ SportDiscus: } & $\begin{array}{l}\text { Actividad física + } \\
\text { adultos mayores }\end{array}$ & 29 & 1 & \multirow{4}{*}{0} & \multirow{4}{*}{0} & \multirow{4}{*}{0} & \multirow{4}{*}{1} \\
\hline & $\begin{array}{l}\text { Adulto mayor + } \\
\text { Salud }\end{array}$ & 14 & 0 & & & & \\
\hline & $\begin{array}{l}\text { Actividad física + } \\
\text { Envejecimiento }\end{array}$ & 10 & 0 & & & & \\
\hline & $\begin{array}{l}\text { Actividad física + } \\
\text { Salud }\end{array}$ & 14 & 0 & & & & \\
\hline $\begin{array}{l}\text { Biblioteca } \\
\text { U.D.C.A: }\end{array}$ & Actividad física & 5 & 0 & 5 & 0 & 0 & 0 \\
\hline \multicolumn{2}{|c|}{ Totales: } & 803 & 15 & \multicolumn{4}{|c|}{20} \\
\hline
\end{tabular}

recopilación de la información, se hace una búsqueda, a través de las bases de datos; posteriormente, una revisión general de los artículos potencialmente funcionales $y$, luego, se resume utilizando el programa Excel, donde se definen los artículos que serán utilizados para el desarrollo del documento; finalmente, se hace una revisión más profunda de los artículos considerados de mayor relevancia.

Revisión de literatura especializada. Para el adecuado desarrollo de la investigación, se hace una exploración en las bases de datos que posee la Universidad de Ciencias Aplicadas y Ambientales U.D.C.A y se limitó al periodo comprendido entre el 2012 y 2018, utilizando las palabras clave actividad física, adulto mayor, salud, envejecimiento y las ecuaciones de búsqueda, que se muestran en la tabla 1. 
Utilizando las anteriores ecuaciones de búsqueda, para encontrar los artículos y documentos relacionados con la temática de actividad física en el adulto mayor, se observa cómo existe una variedad de investigaciones al respecto; no obstante, la cantidad de documentos funcionales, se reduce de manera significativa, además, se debe tener en cuenta, que la cantidad de artículos a nivel regional y nacional es casi nula, a partir de la selección de los veinte artículos que presentan una mayor relación con la temática. Se hace la lectura profunda de estos y se identifican quince artículos funcionales, teniendo en cuenta la temática y los datos obtenidos de los mencionados artículos, como se muestra, posteriormente; se toman los seis mejores artículos de los quince anteriores, debido a que resume, de mejor manera, los ejes temáticos de las investigaciones, y a partir de ellos, se genera una comparación y un análisis detallado en el artículo presente.

\section{MATERIALES Y MÉTODOS}

Se realizó una indagación de artículos relacionados con el tema de actividad física en el adulto mayor y similares, se encontraron ocho artículos de revistas indexadas, que abordaban la temática de actividad física realizada por adultos mayores. Seguidamente, se descartaron dos artículos, debido a que se concentraba en patologías presentadas en la población de adultos mayores, por tanto, se hace una revisión rigurosa de seis artículos sobre el tema de interés. Además, se hizo una revisión de conceptos encontrados en los artículos en la Web, libros y otros autores, para ampliar la perspectiva que se tiene acerca de la actividad física practicada por adultos mayores, las distintas metodologías dadas por los autores en los artículos, se resumen en la tabla 2.

Tabla 2. Metodologías de los estudios consultados.

\begin{tabular}{|c|c|}
\hline Artículo & Metodología \\
\hline $\begin{array}{l}\text { Ambientes } \\
\text { urbanos y } \\
\text { actividad física } \\
\text { en adultos } \\
\text { mayores: } \\
\text { Relevancia del } \\
\text { tema para } \\
\text { América Latina: }\end{array}$ & $\begin{array}{l}\text { Es una revisión literaria acerca de la actividad física, el adulto } \\
\text { mayor, el medio ambiente y los cambios de este en américa } \\
\text { latina, se orienta a entender los vínculos entre ambientes } \\
\text { urbanos y actividad física en adultos mayores, argumenta que en } \\
\text { América Latina no se han generado avances suficientes en el } \\
\text { desarrollo social que le brinden a la población el acceso a servicios } \\
\text { de salud, vivienda y demás aspectos vinculados con su protección } \\
\text { social; también, ambientes urbanos construidos que le faciliten un } \\
\text { envejecimiento activo y funcional, en su descripción aborda los } \\
\text { procesos de urbanización en América Latina y la experiencia en } \\
\text { Bogotá; dice que el crecimiento de las ciudades de la región se } \\
\text { ha caracterizado por ser poco planeado, lo cual propició el } \\
\text { crecimiento de grandes sectores urbanos que presentan } \\
\text { deficiencias notables en su infraestructura física y en los } \\
\text { estándares básicos de desarrollo urbano aunque también } \\
\text { argumenta que a pesar de este crecimiento descontrolado, } \\
\text { ciudades de la región como curitiba y Bogotá, han } \\
\text { implementado cambios urbanos que posiblemente han logrado } \\
\text { tener un efecto positivo en los niveles de actividad física en } \\
\text { todala población, comolo indica Hernández (2010). }\end{array}$ \\
\hline $\begin{array}{l}\text { Motivación auto } \\
\text { determinada en } \\
\text { adultos mayores } \\
\text { practicantes de } \\
\text { ejercicio físico: }\end{array}$ & $\begin{array}{l}\text { El estudio pretendía comprobar la capacidad predictiva de la } \\
\text { valoración del comportamiento autónomo, las necesidades } \\
\text { psicológicas básicas y el índice de autodeterminación sobre los } \\
\text { motivos de práctica auto determinados (finess/salud, social y } \\
\text { disfrute) en adultos mayores. El análisis se le practicó a } 237 \text { adultos } \\
\text { mayores con edades comprendidas entre los } 65 \text { y } 85 \text { años; para el } \\
\text { estudio se midieron cuatro componentes principales: valoración } \\
\text { del comportamiento autónomo, necesidades psicológicas básicas } \\
\text { en el ejercicio, motivación y motivos de práctica para el ejercicio } \\
\text { físico, como resultados más importantes se menciona la } \\
\text { importancia que los factores psicosociales podrían tener en la } \\
\text { práctica de ejercicio físico saludable para el envejecimiento activo } \\
\text { después de la edad adulta, indicado por Marcos Pardo (2014). }\end{array}$ \\
\hline $\begin{array}{c}\text { Actividad física y } \\
\text { salud en el adulto } \\
\text { mayor de seis } \\
\text { países } \\
\text { latinoamericanos: }\end{array}$ & $\begin{array}{l}\text { Es una revisión de literatura cuyo objetivo fue analizar la } \\
\text { situación del adulto mayor en diferentes países } \\
\text { latinoamericanos (Chile, México, Cuba, Colombia, Brasil, } \\
\text { Argentina y Venezuela) además pretendía conocer la relación de } \\
\text { calidad de vida y salud que el ejercicio físico provoca en este } \\
\text { grupo etario y como es percibido por ellos mismos, el articulo } \\
\text { concluye que para el adulto mayor, la actividad física juega un } \\
\text { papel importante en la consecución de un envejecimiento }\end{array}$ \\
\hline
\end{tabular}




\section{Continuación tabla 2.}

\begin{tabular}{|c|c|}
\hline & $\begin{array}{l}\text { exitoso integral, que el principal objetivo que posee este } \\
\text { grupo etario es envejecer con autonomía funcional, } \\
\text { inserto activamente tanto en el núcleo familiar como en la } \\
\text { sociedad y con gran desarrollo del aspecto psicológico y } \\
\text { emocional. Según Roca Moyano (2016): Las necesidades, } \\
\text { objetivos y características de la población adulto mayor en } \\
\text { Latinoamérica presentan gran concordancia entre uno y otro } \\
\text { país. }\end{array}$ \\
\hline $\begin{array}{l}\text { Actividad física } \\
\text { recreativa en el } \\
\text { adulto mayor: }\end{array}$ & $\begin{array}{l}\text { El estudio es descriptivo de enfoque mixto y se centra en un } \\
\text { tipo de estudio evaluativo. La población que hizo parte del } \\
\text { programa "Moviéndonos para vivir mejor", pertenecen al } \\
\text { barrio Panorama de la comuna Macarena de la Ciudad de } \\
\text { Manizales, Caldas, Colombia. Participaron } 19 \text { adultos } \\
\text { mayores entre } 60 \text { y } 80 \text { años; } 18 \text { mujeres y } 1 \text { hombre. El } \\
\text { programa se desarrolló durante } 6 \text { meses interviniendo a la } \\
\text { población tres veces a la semana con una intensidad de una } \\
\text { hora. Las pruebas que se le practicaron al grupo mencionado } \\
\text { fueron: La anamnesis que permitió identificar las } \\
\text { enfermedades que prevalecen en el grupo; la composición } \\
\text { corporal donde se evaluó talla y peso de cada uno de los } \\
\text { participantes del programa para calcular el IMC; la evaluación } \\
\text { condicional, se aplicó la batería SFT (Senior Fitness Test), } \\
\text { diseñada por Rikli \& Jones (2001), al iniciar el programa } \\
\text { (primera prueba) y al final del programa (segunda prueba); la } \\
\text { calidad de vida, aplicando el cuestionario World Health } \\
\text { Organization Quality of Life Questionnaire Group, 1993 } \\
\text { (WHOQOL) (Ruíz Ortega \& Goyes, 2015). }\end{array}$ \\
\hline $\begin{array}{l}\text { La actividad } \\
\text { física para el } \\
\text { adulto mayor en } \\
\text { el medio natural: }\end{array}$ & $\begin{array}{l}\text { Es una revisión de literatura, el artículo realiza un análisis de } \\
\text { la actividad física, sus beneficios y algunos mitos que han } \\
\text { permanecido a lo largo del tiempo en torno al adulto. Realiza } \\
\text { una revisión de los estudios en el ámbito de actividad física } \\
\text { para distintas poblaciones, analizando sus variables } \\
\text { fisiológicas, psicológicas y sociales, tales como el estado de } \\
\text { ánimo, la depresión y la satisfacción que obtienen las } \\
\text { personas al realizar actividad física, el estudio se centra en el } \\
\text { análisis de cuatro puntos: Beneficios del ejercicio en el adulto } \\
\text { mayor, la recreación del adulto mayor, el adulto mayor y su } \\
\text { actividad física en el medio natural, barreras y mitos por } \\
\text { vencer en la población adulta mayor referidos por Jiménez } \\
\text { Oviedo et al. (2013). }\end{array}$ \\
\hline Sin excusas: & $\begin{array}{l}\text { Es un artículo de reporte, que pretende dar una visión } \\
\text { sintetizada acerca de los efectos causados por la actividad } \\
\text { física en el adulto mayor, hace énfasis en que el ejercicio para } \\
\text { los adultos mayores debe recetarse, no sugerirse. Basilio } \\
\text { (2013) comenta: "Hay que dosificar y se deben tomar en } \\
\text { consideración dos parámetros, que no son sexo ni edad, sino } \\
\text { el estado de salud y el grado de capacidad física que } \\
\text { presenten. Con eso se diseñarán programas personalizados", } \\
\text { argumenta que la tercera edad no impide ejercitarse, afirman } \\
\text { expertos que la actividad física brinda múltiples beneficios al } \\
\text { adulto mayor sin importar la edad, la actividad física es crucial } \\
\text { para el organismo. Indica Basilio (2013) que lo importante es } \\
\text { saber qué deportes practicar y dónde hacer ejercicio, en } \\
\text { especial cuando se es un adulto mayor, un individuo de más } \\
\text { de } 60 \text { años. }\end{array}$ \\
\hline
\end{tabular}




\section{RESULTADOS Y DISCUSIÓN}

Por ser estudios variados, se hace conveniente revisar los resultados dados por los investigadores en los artículos de carácter cuantitativo; a continuación, se mencionan los resultados más relevantes encontrados en los distintos estudios (Tabla 3).

Tabla 3. Resultados de los estudios consultados.

\begin{tabular}{|c|c|}
\hline Artículo & Resultados \\
\hline $\begin{array}{c}\text { Motivación } \\
\text { autodeterminada } \\
\text { en adultos } \\
\text { mayores } \\
\text { practicantes de } \\
\text { ejercicio físico: }\end{array}$ & $\begin{array}{l}\text { La valoración del comportamiento autónomo presentó una } \\
\text { media de 4.68. El mediador psicológico más valorado fue el de } \\
\text { relación con los demás, le sigue la autonomía y la competencia } \\
\text { percibida. El índice de autodeterminación osciló de }-10.17 \text { a } \\
\text { 13.92, con un valor medio de } 9 \text {. El motivo de práctica deportiva } \\
\text { fitness/salud obtuvo mayor puntuación que el motivo disfrute y el } \\
\text { motivo social. En el análisis de correlación se observó que la } \\
\text { variable valoración del comportamiento autónomo } \\
\text { correlacionó positivamente con la autonomía y con la relación con } \\
\text { los demás Marcos Pardo (2014) define las tres } \\
\text { necesidades psicológicas cósicas correlacionaron } \\
\text { positivamente entre sí, con el índice de autodeterminación y con } \\
\text { los motivos de práctica autodeterminados. }\end{array}$ \\
\hline $\begin{array}{l}\text { Actividad física } \\
\text { recreativa en el } \\
\text { adulto mayor: }\end{array}$ & $\begin{array}{l}\text { De acuerdo con los resultados obtenidos se concluye que el } \\
\text { programa de actividad física realizado durante } 6 \text { meses ( } 3 \\
\text { veces por semana) influyó positivamente en el incremento de } \\
\text { fuerza, equilibrio, resistencia y flexibilidad. La prueba de } \\
\text { resistencia es la de mayor puntuación seguida de la prueba de fuerza } \\
\text { (miembros superiores e inferiores). El programa de actividad } \\
\text { física a través de actividades jugadas mejora la condición física } \\
\text { de los participantes, en cuanto a calidad de vida (WHOQOL); en } \\
\text { la primera evaluación las personas afirman sentirse bien con } \\
\text { su calidad de vida, a través del programa de actividad física y } \\
\text { diversas actividades se observa un cambio muy significativo el } 42,1 \% \\
\text { afirma sentirse bastante bien y el } 36,8 \% \text { responde estar muy bien; } \\
\text { tan solo el } 21,1 \% \text { afirma sentirse normal (Ruíz Ortega \& Goyes, } \\
2015 \text { ). }\end{array}$ \\
\hline Sin excusas: & $\begin{array}{l}\text { Beneficios qué aporta el ejercicio a los adultos mayores } \\
\text { (Basilio, 2013): } \\
\text { Emocionales: } \\
\text {-Mejora la actividad mental, así como la coordinación y el } \\
\text { equilibrio. } \\
\text {-Combate el estrés y la ansiedad. } \\
\text {-Provee un mejor estilo de vida. } \\
\text {-Aporta diversión. } \\
\text {-Incide en el estado de ánimo. } \\
\text {-Permite a las personas sentirse útiles e independientes. } \\
\text {-Ayuda a combatir el insomnio. } \\
\text { Físicos: } \\
\text {-Salud en general. } \\
\text {-Disminuye el riesgo de desarrollar enfermedades. } \\
\text {-Interviene en el buen funcionamiento cardiovascular. } \\
\text {-Minimiza la pérdida de masa muscular y ósea. } \\
\text {-Aumenta la flexibilidad. } \\
\text {-Mejora la coordinación motriz. } \\
\text {-Ayuda a mantener el peso. }\end{array}$ \\
\hline
\end{tabular}


Debido a que existen artículos de revisión literaria, no se puede hacer una relación entre los estudios netamente cuantitativos, como se haría con los artículos que manejan un método de estudio con base al análisis de datos; aun así, se puede hacer una comparación entre los seis artículos, teniendo en cuenta el aspecto cualitativo, según los resultados y conclusiones de los autores.

Cabe resaltar que los seis artículos hacen énfasis en mostrar los beneficios que conlleva la práctica de actividad física, por parte de la población de adultos mayores; el artículo Ambientes urbanos y actividad física en adultos mayores: Relevancia del tema para América Latina y en el artículo Actividad física y salud en el adulto mayor de seis países latinoamericanos, hace una crítica a las políticas públicas con relación a la actividad física y la salud de los adultos mayores, mencionando que es necesario tomar mayores acciones para conseguir una vejez activa y saludable en sus pobladores, específicamente, en la población de adultos mayores.

Por otra parte, los otros cuatro artículos revisados hacen hincapié en los beneficios sobre la salud física, mental y emocional, que conlleva la realización de actividad física, de forma frecuente, en poblaciones de adultos mayores. Es imperativo resaltar el artículo La actividad física para el adulto mayor en el medio natural y, sin excusas, llegan a conclusiones similares, donde recomiendan que la actividad física realizada por el adulto mayor, se debe realizar en sitios abiertos y en el medio ambiente, de ser posible, recomendando sitios, como parques, bosques zonas rurales y afines. Conjuntamente, los autores consultados, mencionan que la actividad física realizada por adultos mayores, de una manera guiada y frecuente, permite una mejora o un aumento en la calidad de vida de los participantes. Además de eso, mencionan algunos beneficios de la actividad física realizada por los adultos mayores, que presenten algún tipo de patología.

\section{CONCLUSIONES}

Se toman los conclusivos más relevantes dados por los autores de los seis artículos revisados, Basilio (2013); Hernández et al. (2010); Marcos Pardo et al. (2014); Roca Moyano (2016); Ruíz Ortega \& Goyes (2015); Jiménez Oviedo et al. (2013), que se resume en la tabla 4.

Tabla 4. Conclusiones de los estudios consultados.

\begin{tabular}{|c|c|}
\hline Artículo & Conclusiones \\
\hline $\begin{array}{c}\text { Ambientes } \\
\text { urbanos y } \\
\text { actividad física } \\
\text { en adultos } \\
\text { mayores: } \\
\text { Relevancia del } \\
\text { tema para } \\
\text { América Latina: }\end{array}$ & $\begin{array}{l}\text { Para América Latina no se han generado avances suficientes en el } \\
\text { desarrollo social, que le brinden a la población de adultos mayores } \\
\text { ambientes urbanos construidos que le faciliten un } \\
\text { envejecimiento activo y funcional. } \\
\text { Para Hernández (2010) la evidencia revisada en este artículo } \\
\text { sugiere que los ambientes urbanos construidos están } \\
\text { asociados con la salud de los adultos mayores, } \\
\text { específicamente en actividad paderica. } \\
\text { Un desafío mayor para la sociedad y sus tomadores de } \\
\text { decisiones es reconocer que una vejez activa está } \\
\text { determinada, en parte, por la manera en que se ha } \\
\text { desarrollado el ciclo vital de cada persona. }\end{array}$ \\
\hline $\begin{array}{l}\text { Motivación auto } \\
\text { determinada en } \\
\text { adultos mayores } \\
\text { practicantes de } \\
\text { ejercicio físico: }\end{array}$ & $\begin{array}{l}\text { Marcos Pardo (2014) expone los motivos de práctica social y } \\
\text { disfrute fueron predichos positivamente por la valoración del } \\
\text { comportamiento autónomo, la competencia percibida y la } \\
\text { relación con los demás, aunque se relacionaron } \\
\text { positivamente con las tres necesidades psicológicas básicas } \\
\text { (autonomía, competencia y relación con los demás) y con el } \\
\text { índice de autodeterminación. Su conclusión dice, "el estudio } \\
\text { podría servir a los técnicos deportivos para que conozcan la } \\
\text { importancia de valorar la actitud de los practicantes y saber de la } \\
\text { importancia de utilizar estrategias motivacionales que garanticen } \\
\text { la satisfacción de las necesidades psicológicas básicas. }\end{array}$ \\
\hline
\end{tabular}


Continuación tabla 4.

Actividad física y salud en el adulto mayor de seis países latinoamericanos:

Actividad física recreativa en el adulto mayor: para el adulto mayor en el medio natural:

Sin excusas:
Se aprecia que en general, los adultos mayores latinoamericanos presentan homogeneidad tanto en características sociales, necesidades biológicas y funcionales, como en objetivos a cumplir durante la vejez. Se pueden apreciar ejes, características y objetivos comunes entre ellos.

Si bien existe evidencia científica, el tema del envejecimiento poblacional latinoamericano es contemporáneo y que demanda aún más esfuerzos en las políticas públicas de los diferentes países, a fin de brindar a las personas mayores un envejecimiento activo, saludable e integral.

Roca Moyano (2015) opina que, en Latinoamérica, los adultos mayores presentan similitudes tanto en necesidades a satisfacer como en objetivos a lograr durante esta etapa de vida, debido a que en general, el adulto mayor desea tener una vejez con autonomía funcional.

El trabajo realizado con el adulto mayor redujo el peso corporal, mejoró la condición física; logrando vincular a este tipo de población en actividades recreativas que le hicieran ser protagonista activo de una calidad de vida adecuada. El trabajo realizado ayudó para que el adulto mayor sea cada vez más autónomo e independiente en ciertas circunstancias. A través de la recreación se logra una adecuada transposición didáctica con relación a la rutinaria forma de trabajar con este tipo de población.

Para Ruíz Ortega \& Goyes (2015) el rol del profesor es valorado en tanto es un motivador constante y con calidad humana.

Los beneficios se perciben principalmente en los factores fisiológicos, psicológicos y sociales. Sin embargo, estos beneficios dependerán estrictamente de cada individuo y por supuesto, del trabajo planeado y del que se anhela con éste, según planificación del profesional, adaptada a las particularidades. La recreación al aire libre es la invitación.

Jiménez Oviedo (2013) menciona: Convivir amigablemente en este espacio es una herramienta diferente; puede ser un trabajo muy enriquecedor, puesto que la naturaleza beneficia al ser humano a través de su visita y su cuidado.

Hace referencia que la actividad física se debe practicar al aire libre, de ser posible y en sitios como gimnasios, Basilio (2013) comenta: "a todos les ponen exactamente lo mismo (de rutina) y no debe ser así. No se deben repetir las acciones del gimnasio porque se corren riesgos de lesiones o peor aún, de un ataque cardíaco, debido a que los instructores no conocen las enfermedades crónicas que padece la persona, como hipertensión, alteración de los lípidos, diabetes, entre otros". Por último, Basilio (2013) menciona que los beneficios que aporta el ejercicio a los adultos mayores se dividen en dos: Emocionales y físicos. 
Realizada la revisión de los artículos y el análisis de sus diferentes conclusivos, se demuestra que la actividad física trae beneficios en la salud física y emocional de los adultos mayores, ayudando a mitigar el riesgo de desarrollar enfermedades crónicas no transmisibles, además de traer beneficios psicológicos, sociales y fisiológicos, a quienes practican la actividad física de forma frecuente y de manera orientada.

Es importante estudiar el tema, debido a que, muchas veces, el adulto mayor no es tenido en cuenta, como parte significativa dentro de la sociedad; además, por lo general, el profesional en Ciencias del Deporte, se enfoca en trabajar el deporte de alto rendimiento o en población joven, dejando a un costado los compromisos con poblaciones de adultos mayores; de esta manera, se generan otras opciones de ocupación laboral.

\section{REFERENCIAS}

1. BASILIO, A. 2013. Sin excusas. Monterrey. El sol S.A.

\section{CUESTIONARIO CALIDAD DE VIDA WHOQOL GROUP.} 1993. Study Protocol for the World Health Organization Project to develop a Quality of Life Assessment Instrument (WHOQOL) Qual Life Res. 2:153-159.

3. DULCEY-RUÍZ, E. 2010. Psicología social del envejecimiento y perspectiva del transcurso de la vida: consideraciones críticas. Revista Colombiana de Psicología. 19(2):207-224.

4. GÓMEZ, F.; MATEUS, J.C.; CABRERA, G. 2004. Leisure-time physical activity among women in a neighbourhood in Bogotá, Colombia: prevalence and socio-demographic correlates. Cuadernos de Salud Pública. 20(4):1103-1109.

5. HERNÁNDEZ, A.; GÓMEZ, L.F.; PARRA, D.C. 2010. Ambientes urbanos y actividad física en adultos mayores: Relevancia del tema para América Latina. Rev. Salud pública. 12(2):327-335.

6. HERNÁNDEZ SAMPIERI, R.; FERNANDEZ COLLADO, C.; BAPTISTA LUCIO, M. DEL P. 2014. Metodología de la investigación. Sexta edición. Ed. Mc Graw Hill. México, D.F. 634p.
7. JACOBY, E.; BULL, F.; NEIMAN, A. 2003. Cambios acelerados del estilo de vida obligan a fomentar la actividad física como prioridad en la región de las Américas. Revista Panam Salud Pública. 14(4):223-225.

8. JIMENEZ OVIEDO, Y.; NÚNEZ, M.; COTO, E. 2013. La actividad física para el adulto mayor en el medio natural. InterSedes. 14(27):68-181.

9. LOBELO, F. 2006. Carga de mortalidad asociada a la inactividad física en Bogotá. Rev Salud Pública (Bogotá). 8:28-41.

10. MARCOS PARDO, P.J.; ORQUIN CASTRILLON, F.J.; BELANDO PEDRENO, N.; MORENO-MURCIA, J.A. 2014. Motivación autodeterminada en adultos mayores practicantes de ejercicio físico. Cuadernos de Psicología del Deporte. 14(3):149-156.

11. MONTEIRO, A.; CONDE, W.; MATSUDO, S.; MATSUDO, V.; BONSEÑOR, I.; LOTUFO, P. 2003. Descriptive epidemiology of leisuretime physical activity in Brazil, 1996-1997. Rev Panam Salud Pública. 14(4):246-254.

12. ORGANIZACIÓN MUNDIAL DE LA SALUD, OMS. 2002. Promover un estilo de vida para mayores. Guía regional para la promoción de la actividad física. Disponible en: http:// www.imsersomayores.csic.es/documentos/ documentos/ops-promover-01.pdf (con acceso el 04/2019).

13. ORGANIZACIÓN PANAMERICANA DE LA SALUDOPS. 2002. La inactividad física: un factor de riesgo para la salud en las Américas. Programa de Alimentación y nutrición/ División de promoción y protección de la salud. Disponible en: www.ops-oms.org/ Spanish/HPP/HPN/whd2002-factsheet3.pdf (con acceso el 04/2019).

14. RIKLI, R.; JONES, C. 2001. Senior fitness test manual. Estados Unidos de América. Human Kinetics.

15. ROCA MOYANO, R.E. 2016. Actividad Física y Salud en el adulto mayor de 6 países latinoamericanos: review. Revista de Ciencias de la Actividad Física UCM. 17(1):77-86. 
16. RUÍZ ORTEGA, O.; GOYES, E. 2015. Actividad física recreativa en el adulto mayor. Educación Física y Deporte. 34(1):239-267. https://doi. org/10.17533/udea.efyd.v34n1a11 